\title{
Sphingosine Induces Apoptosis in MKN-28 Human Gastric Cancer Cells in an SDK-Dependent Manner
}

\author{
Takeshi Kanno ${ }^{a}$ Takaaki Nishimoto ${ }^{a}$ Yumiko Fujita ${ }^{a, b}$ Akinobu Gotoh ${ }^{c}$ Takashi
} Nakano $^{b}$ Tomoyuki Nishizaki $^{\mathrm{a}}$

aDivision of Bioinformation, Department of Physiology, Hyogo College of Medicine, 1-1 Mukogawacho, Nishinomiya; ${ }^{b}$ Department of Thoracic Oncology, Hyogo College of Medicine, 1-1 Mukogawa-cho, Nishinomiya; 'Laboratory of Cell and Gene Therapy, Institute for Advanced Medical Sciences, Hyogo College of Medicine, 1-1 Mukogawa-cho, Nishinomiya

\section{Key Words}

Sphingosine $\cdot$ Protein kinase $C-\delta \cdot$ Sphingosine-dependent protein kinase $\bullet$ Gastric cancer cell - Apoptosis

\begin{abstract}
Background/Aims: Evidence has pointed to the role of sphingosine in cellular differentiation, cell growth, and apoptosis. The present study investigated sphingosine-induced apoptosis in human gastric cancer cells. Methods: Well differentiated MKN-28 and poorly differentiated MKN-45 human gastric cancer cells were cultured. MTT assay, TUNEL staining, Western blotting, and assay of caspase-3, -8, and -9 activities were carried out in cells transfected with and without the siRNA to silence the protein kinase C (PKC)- $\delta$-targeted gene. Results: Sphingosine induced apoptosis in MKN-28 cells, with the potential much greater than for MKN-45 cells. Transfection with the siRNA to silence the PKC- $\delta$-targeted gene (PKC- $\delta$ siRNA) into $\mathrm{MKN}-28$ cells significantly reduced presence of sphingosine-dependent protein kinase (SDK) in association with reduced PKC- $\delta$ expression. Sphingosine-induced apoptosis in MKN-28 cells was prevented by transfecting with the PKC- $\delta$ siRNA. Sphingosine promoted SDK production from PKC- $\delta$ and increased phosphorylated 14-3-3 protein for MKN-28 cells, but such effects were not found with MKN-45 cells. Moreover, sphingosine perturbed mitochondrial membrane potentials and activated caspase- 3 and caspase- 9 in MKN-28 cells, which were also inhibited by transfecting with the PKC- $\delta$ siRNA. Conclusion: The results of the present study indicate that sphingosine induces apoptosis in well differentiated MKN-28 human gastric cancer cells by increasing SDK production from PKC- $\delta$, to phosphorylate 14-33 protein, thereby causing disruption of mitochondrial membrane potentials and activating caspase- 9 followed by the effector caspase-3.
\end{abstract}

Copyright $(2012$ S. Karger AG, Basel 
Kanno/Nishimoto/Fujita et al.: Sphingosine-Induced MKN-28 Cell Apoptosis

\section{Introduction}

Sphingolipids such as ceramide, ceramide 1-phosphate, sphingosine, and sphingosine 1-phosphate (S1P) are implicated in the regulation of cellular differentiation, cell growth, and apoptosis. Mounting evidence has pointed to the role of ceramide in apoptosis via diverse signaling pathways. Sphingosine, that is produced from ceramidase-catalyzed cleavage of fatty acids from ceramide, also participates in apoptotic cell death. Sphingosine induces apoptosis in rhabdomyosarcoma cells through mitochondrial Bax and the ensuing caspase activation [1]. Sphingosine, alternatively, induces apoptosis in mouse BALB/c 3T3 clone A31 cells via an SDK pathway [2]. Furthermore, sphingosine induces apoptosis in hippocampal neurons and astrocytes by activating caspase-3/-9 via an SDK/14-3-3 protein/ Bax/cytochrome c pathway [3]. SDK is a protein kinase that is produced through proteolytic processing of PKC- $\delta$ and it is activated by binding sphingosine [2]. SDK phosphorylates 14-33 protein as a target $[4,5]$, which could dissociate Bax from a complex with 14-3-3 protein, to induce a mitochondria-dependent apoptosis.

The present study aimed at understanding sphingosine-induced apoptosis in human gastric cancer cells and the underlying pathway. We show here that sphingosine induces apoptosis in well differentiated MKN-28 human gastric cancer cells by activating caspase3/-9 in concert with mitochondrial damage in an SDK-dependent manner.

\section{Materials and Methods}

Cell cultures

MKN-28 cells, a well differentiated human gastric cancer cell line, and MKN-45 cells, a poorly differentiated human gastric cancer cell line, were grown in a RPMI1640 solution (Sigma, St. Louis, MO, USA) supplemented with $10 \%(\mathrm{v} / \mathrm{v})$ fetal bovine serum, penicillin (final concentration, $100 \mathrm{U} / \mathrm{ml}$ ), and streptomycin (final concentration, $0.1 \mathrm{mg} / \mathrm{ml}$ ) in a humidified atmosphere of $5 \% \mathrm{CO}_{2}$ and $95 \%$ air at $37^{\circ} \mathrm{C}$.

Assay of cell viability

Cell viability was assayed by the method using 3-(4,5-dimethyl-2-thiazolyl)-2,5-diphenyl-2Htetrazolium bromide (MTT) as described previously [3]. MTT-reactive cells were quantified at an absorbance of $570 \mathrm{~nm}$ using a micro-plate reader (SPECTRAmax PLUS384, Molecular Devices, Sunnyvale, CA, USA). Various concentrations of sphingosine were dissolved in ethanol and further, diluted at 1:1000 with culture medium containing MTT when applied. We have confirmed that $0.1 \%(\mathrm{v} / \mathrm{v})$ ethanol has no effect on cell viability for MKN-28 and MKN-45 cells.

Terminal deoxynucleotidyl transferase-mediated dUTP nick end labeling (TUNEL) staining

TUNEL staining was performed to detect in situ DNA fragmentation as a marker of apoptosis using an In Situ Apoptosis Detection Kit (Takara Bio, Otsu, Japan). Briefly, fixed and permeabilized cells were reacted with terminal deoxynucleotidyl transferase and fluorescein isothiocyanate (FITC)-deoxyuridine triphosphate for $90 \mathrm{~min}$ at $37^{\circ} \mathrm{C}$. FITC signals were visualized with a confocal scanning laser microscope (LSM 510, Carl Zeiss Co., Ltd., Oberkochen, Germany).

\section{Monitoring of mitochondrial membrane potentials}

Mitochondrial membrane potentials were measured using a DePsipher ${ }^{\mathrm{TM}}$ kit. Cells untreated and treated with sphingosine were incubated in a DePsipher ${ }^{\mathrm{TM}}$ solution at $37{ }^{\circ} \mathrm{C}$ for $20 \mathrm{~min}$. Then, cells were washed with $1 \mathrm{ml}$ of a reaction buffer containing a stabilizer solution. The fluorescent signals were observed with a laser scanning microscope (LSM 510) equipped with an epifluorescence device using a fluorescein long-pass filter (fluorescein and rhodamine) at an absorbance of $590 \mathrm{~nm}$ for red aggregations and at an absorbance of $530 \mathrm{~nm}$ for green aggregations. 
Fig. 1. Sphingosine-induced MKN28 cell death in a PKC- $\delta$-dependent manner. (A) MKN-28 and MKN-45 cells were treated with sphingosine at concentrations as indicated for $48 \mathrm{~h}$, and then, MTT assay was carried out. In the graph, each point represents the mean $( \pm$ SEM) percentage of control (MTT intensities before treatment with sphingosine $)(\mathrm{n}=4$ independent experiments). (B) MKN-28 cells were treated with sphingosine $(100 \mu \mathrm{M})$ for $48 \mathrm{~h}$ in the absence and presence of GF109203X (+GF)(100 nM). In the graph, each column represents the mean ( \pm SEM) percentage of control (MTT intensities before treatment with sphingosine in the absence of GF109203X)(n=4 independent experiments). (C) MKN-28 cells were transfected with the NC siRNA or the PKC- $\delta$ siRNA, and $48 \mathrm{~h}$ after transfection Western blotting was carried out. Signal intensities for PKC- $\delta$ and SDK proteins were normalized by $\beta$-actin signal

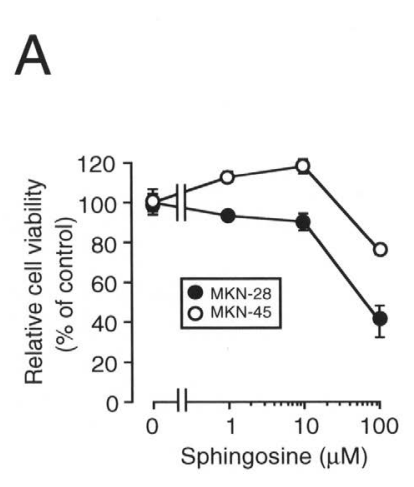

B MKN-28 cell

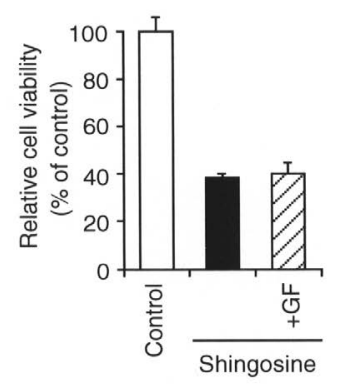

C MKN-28 cell

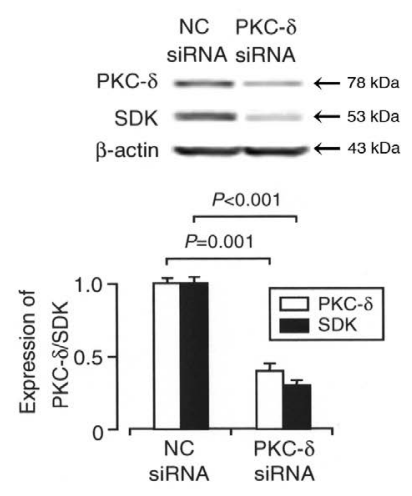

D MKN-28 cell

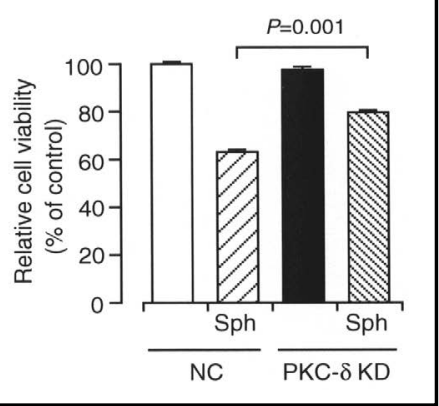

intensities. In the graph, each column represents the mean ( \pm SEM) protein intensity $(n=4$ independent experiments). $P$ values, unpaired $t$-test. (D) MKN-28 cells transfected with the NC siRNA (NC) or the PKC- $\delta$ siRNA (PKC- $\delta$ KD) were treated with sphingosine $(\mathrm{Sph})(100 \mu \mathrm{M})$ for $48 \mathrm{~h}$ followed by MTT assay. In the graph, each column represents the mean \pm SEM) percentage of control (MTT intensities before treatment with sphingosine)(n=4 independent experiments). $P$ values, Dunnett's test.

\section{Enzymatic assay of caspase-3, -8, and -9 activities}

Caspase activity was measured using a caspase fluorometric assay kit (Ac-Asp-Glu-Val-Asp-MCA for a caspase-3 substrate peptide; Ac-Ile-Glu-Thr-Asp-MCA for a caspase-8 substrate peptide; and Ac-Leu-GluHis-Asp-MCA for a caspase-9 substrate peptide) as previously described [3]. Briefly, cells were harvested before and after treatment with sphingosine, and then centrifuged at 3,000 rpm for $5 \mathrm{~min}$ at $4{ }^{\circ} \mathrm{C}$. The pellet was incubated on ice in cell lysis buffer for $10 \mathrm{~min}$, and reacted with the fluorescently labeled tetrapeptide at $37^{\circ} \mathrm{C}$ for $2 \mathrm{~h}$. The fluorescence was measured at an excitation of wavelength of $380 \mathrm{~nm}$ and an emission wavelength of $460 \mathrm{~nm}$ with a fluorometer (Fluorescence Spectrometer, F-4500, HITACHI, Tokyo, Japan).

\section{Construction and transfection of siRNA}

The siRNA to silence PKC- $\delta$-targeted gene (PKC- $\delta$ siRNA) and the negative control siRNA (NC siRNA) were obtained from BONAC (Fukuoka, Japan). The PKC- $\delta$ siRNA and the NC siRNA were reverse-transfected into cells using a Lipofectamine reagent (Invitrogen, Carlsbad, CA, USA). Cells were used for experiments 48 $\mathrm{h}$ after transfection.

\section{Western blotting}

Cells were lysed with $1 \%(\mathrm{w} / \mathrm{v}$ ) sodium dodecyl sulfate (SDS). Proteins were separated by SDSpolyacrylamide gel electrophoresis (SDS-PAGE) using a TGX gel (BioRad, Hercules, CA, USA) and then transferred to polyvinylidene difluoride membranes. Blotting membranes were blocked with TBS-T [150 $\mathrm{mM} \mathrm{NaCl}, 0.1 \%(\mathrm{v} / \mathrm{v})$ Tween 20 and $20 \mathrm{mM}$ Tris, $\mathrm{pH}$ 7.5] containing 5\% (w/v) bovine serum albumin and 
Fig. 2. Sphingosine-induced $\mathrm{MKN}-28$ cell apoptosis in a PKC- $\delta$-dependent manner. MKN-28 cells transfected with the NC siRNA $(\mathrm{NC})$ or the PKC- $\delta$ siRNA (PKC- $\delta \mathrm{KD}$ ) were treated with sphingosine (Sph) $(100 \mu \mathrm{M})$ for $48 \mathrm{~h}$, and then, TUNEL staining was carried out. TUNEL-positive cells were counted in the area $(0.4 \mathrm{~mm} \times 0.4 \mathrm{~mm})$ selected at random. Bars, $50 \mu \mathrm{m}$. In the graph, each column represents the mean $( \pm$ SEM) TUNEL-positive cell percentage of whole cells ( $\mathrm{n}=4$ independent experiments). $P$ values, Dunnett's test.
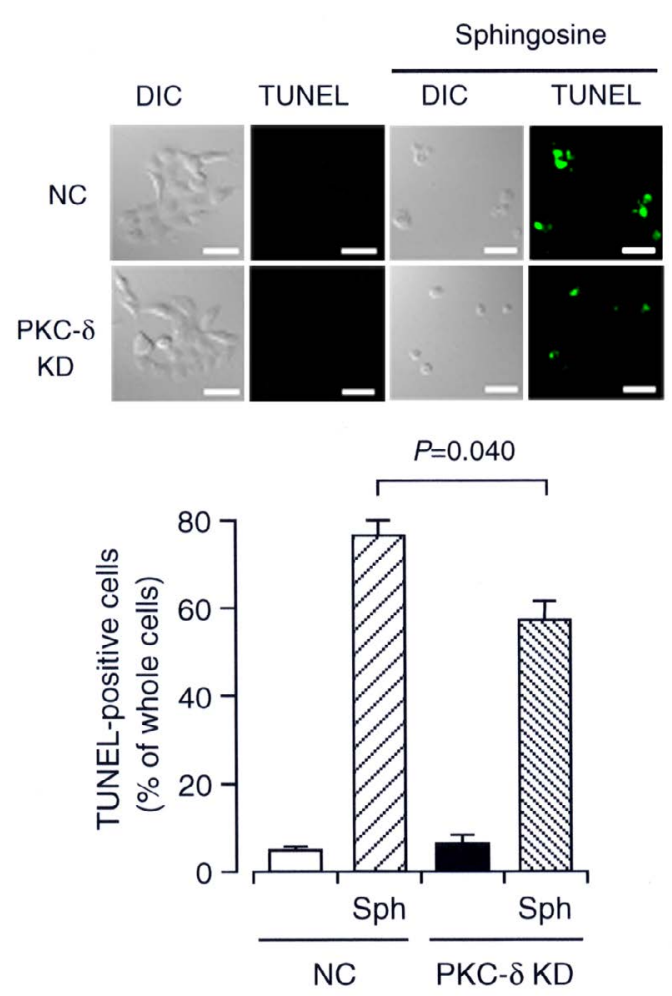

subsequently incubated with an anti-PKC- $\delta$ antibody (Santa Cruz Biotechnology, Santa Cruz, CA, USA), an anti-14-3-3 protein antibody (Abgent, San Diego, CA, USA), an anti-phospho-14-3-3 protein antibody (Abcam, Cambridge, MA, USA), that detects at phospho-Ser58 on 14-3-3 $\gamma, \eta$, and $\zeta$, or an anti- $\beta$-actin antibody (Sigma). After washing, membranes were reacted with a horseradish peroxidase-conjugated goat anti-mouse IgG or goat anti-rabbit IgG antibody. Immunoreactivity was detected with an ECL kit (GE Healthcare, Piscataway, NJ, USA) and visualized using a chemiluminescence detection system (GE Healthcare). Immunoblotting for SDK was carried out using an anti-PKC- $\delta$ antibody (Santa Cruz Biotechnology). Protein concentrations for each sample were determined with a BCA protein assay kit (Pierce, Rockford, IL, USA).

\section{Statistical analysis}

Statistical analysis was carried out using unpaired $t$-test and Dunnett's test.

\section{Results}

Sphingosine induces MKN-28 cell apoptosis in an SDK-dependent manner

For MKN-28 cells, sphingosine reduced cell viability at concentrations more than 10 $\mu \mathrm{M}$, reaching $30 \%$ of untreated basal levels at $100 \mu \mathrm{M}$ (Fig. 1A). In contrast, sphingosine reduced MKN-45 cell viability to much lesser extent (Fig. 1A). For MKN-28 cells, sphingosine $(100 \mu \mathrm{M})$ increased TUNEL-positive cells to $75 \%$ of total cells from $5 \%$ before treatment (Fig. 2). This accounts for sphingosine-induced apoptosis in MKN-28 cells. Sphingosine is metabolized into S1P through its phosphorylation due to sphingosine kinase. S1P at concentrations ranging from 1 to $100 \mu \mathrm{M}$ had no effect on cell viability for MKN-28 and MKN-45 cells (data not shown). This indicates that the sphingosine effect obtained here is not caused by its metabolite S1P.

Sphingosine $(100 \mu \mathrm{M})$-induced reduction in MKN-28 cell viability was not affected by GF109203X (100 nM), an inhibitor of PKC (Fig. 1B). This suggests that sphingosine induces MKN-28 cell apoptosis via a pathway independent of PKC activation. 
Fig. 3. Sphingosine-induced increase in SDK production. Western blotting for PKC- $\delta$ and SDK was carried out in MKN-28 (A) and MKN-45 cells (B) before and after treatment with sphingosine $(100 \mu \mathrm{M})$. Signal intensities for PKC- $\delta$ and SDK proteins were normalized by $\beta$-actin signal intensities. In the graphs, each point represents the mean $( \pm$ SEM) protein intensity $(n=4$ independent experiments).

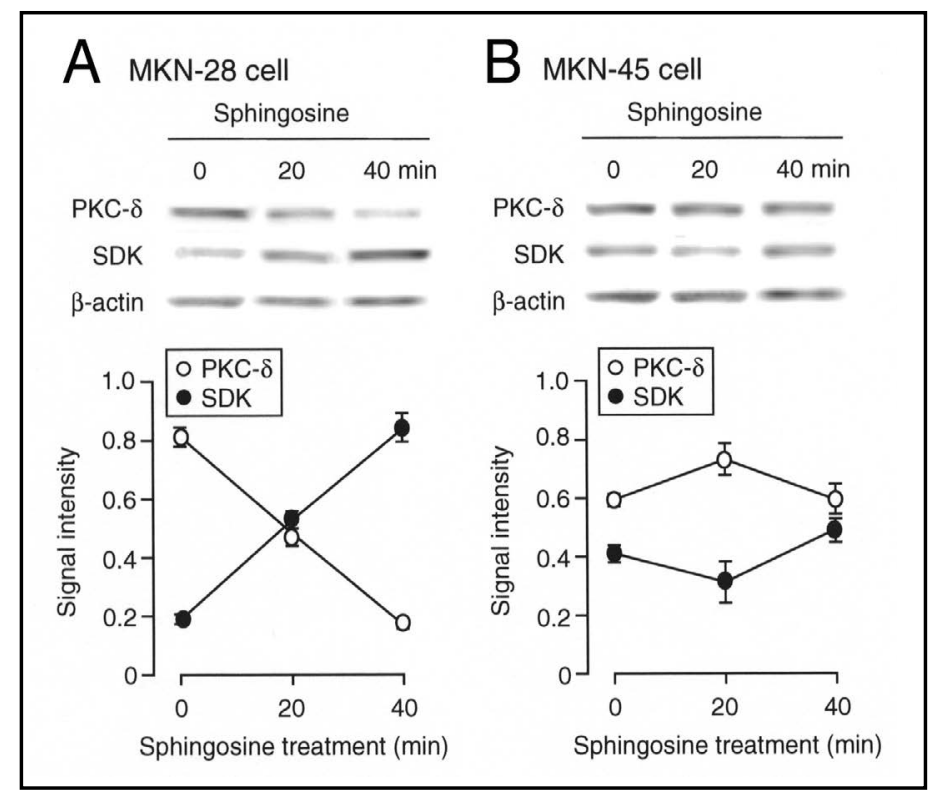

Fig. 4. Sphingosine-induced phosphorylation of 14-3-3 protein. Western blotting for 14-3-3 protein $\zeta$ and phospho-14-3-3 protein (P-143-3 protein) was carried out in MKN28 (A) and MKN-45 cells (B) before and after treatment with sphingosine $(100 \mu \mathrm{M})$. Signal intensities for 143-3 protein $\zeta$ and phospho-14-3-3 protein were normalized by $\beta$-actin signal intensities. In the graphs, each point represents the mean $( \pm$ SEM) protein intensity $(n=4$ independent experiments).

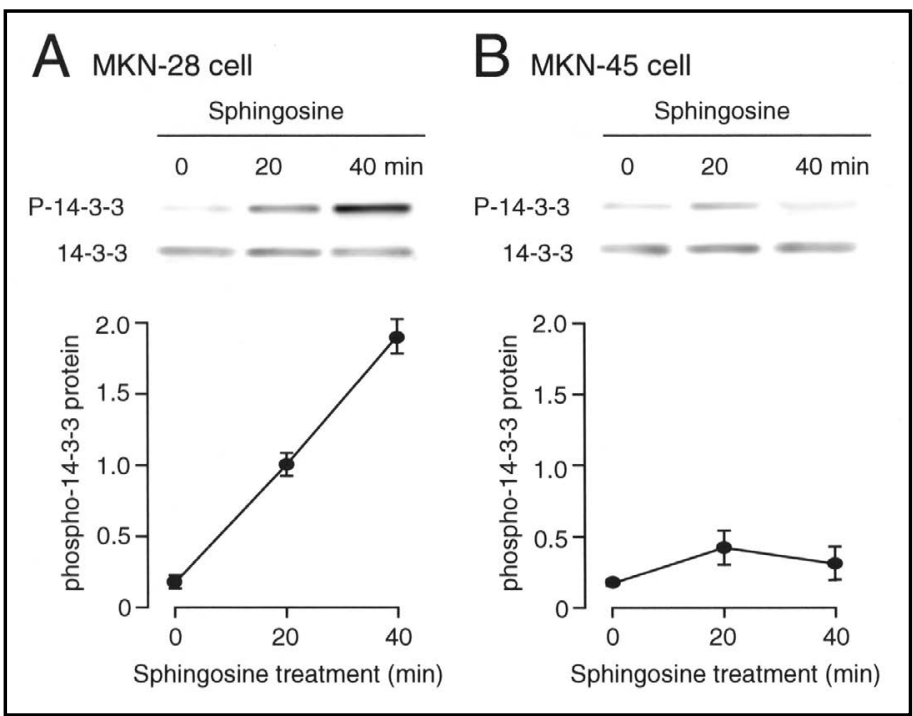

Sphingosine is recognized to activate SDK, that is produced through proteolytic processing of PKC- $\delta$ [2]. To examine the implication of PKC- $\delta /$ SDK in sphingosine-induced MKN-28 cell apoptosis, we have constructed the PKC- $\delta$ siRNA. Expression of PKC- $\delta$ protein was significantly decreased in MKN-28 cells transfected with the PKC- $\delta$ siRNA as compared with the expression for cells transfected with the NC siRNA (Fig. 1C), which confirms PKC- $\delta$ knocking-down. Notably, SDK presence was also suppressed by transfecting with the PKC- $\delta$ siRNA (Fig. 1C). Sphingosine-induced reduction in MKN-28 cell viability or an increase in TUNEL-positive cells was significantly prevented by transfecting with the PKC- $\delta$ siRNA (Fig. $1 D, 2)$. This, in the light of the fact that the sphingosine effect on MKN-28 cell viability was independent of PKC activation, suggests that sphingosine induces MKN-28 cell apoptosis by activating SDK.

Sphingosine increases SDK production from PKC- $\delta$ and phosphorylates 14-3-3 protein in MKN-28 cells

Sphingosine $(100 \mu \mathrm{M})$ increased the amount of SDK in parallel with decreased PKC- $\delta$ in a treatment time (0-40 min)-dependent manner for MKN-28 cells (Fig. 3A). In contrast, 


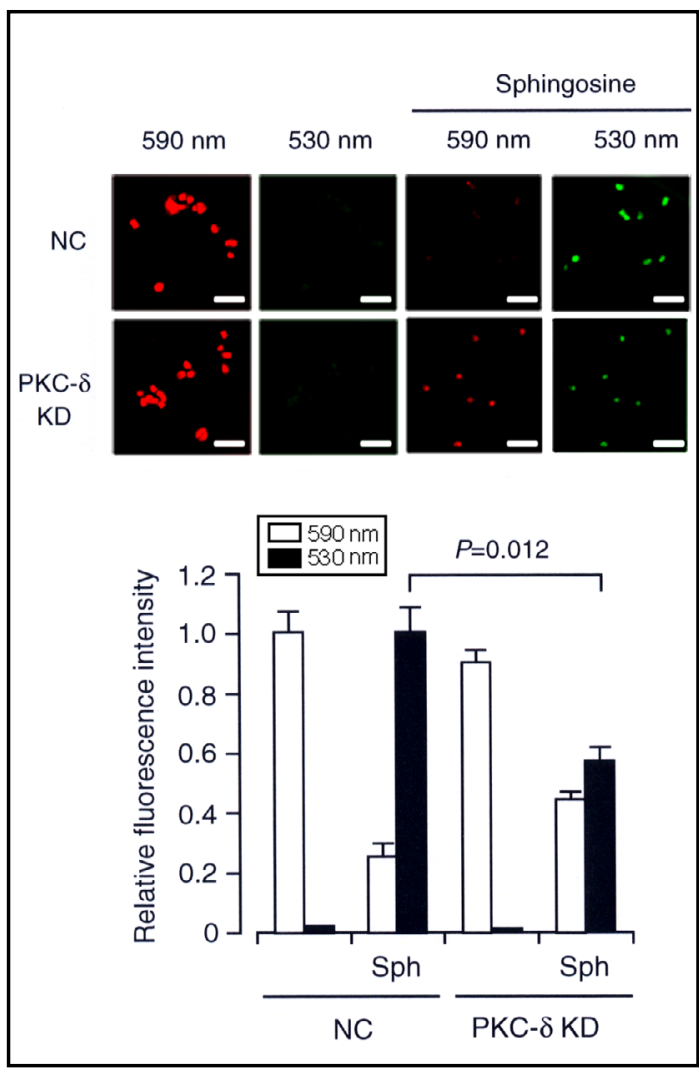

Fig. 5. Sphingosine-induced disruption of mitochondrial membrane potentials in gastric cancer cells. Mitochondrial membrane potentials were monitored in MKN-28 cells transfected with the NC siRNA (NC) or the PKC- $\delta$ siRNA (PKC- $\delta$ KD) before and after 1-h treatment with sphingosine $(100 \mu \mathrm{M})$. Typical fluorescent images are shown in the upper panel. Bars, $50 \mu \mathrm{m}$. Red and green fluorescent intensities in the area $(0.4 \mathrm{~mm} \times 0.4$ $\mathrm{mm}$ ) selected at random were measured using ImageJ (Bethesda, MD, USA), and red fluorescent intensities at an absorbance of $590 \mathrm{~nm}$ for cells untreated with sphingosine or green fluorescent intensities at an absorbance of $530 \mathrm{~nm}$ for cells treated with sphingosine were regarded as 1 . In the graph, each column represents the mean $( \pm$ SEM) intensity (n=4 independent experiments). $P$ value, Dunnett's test.
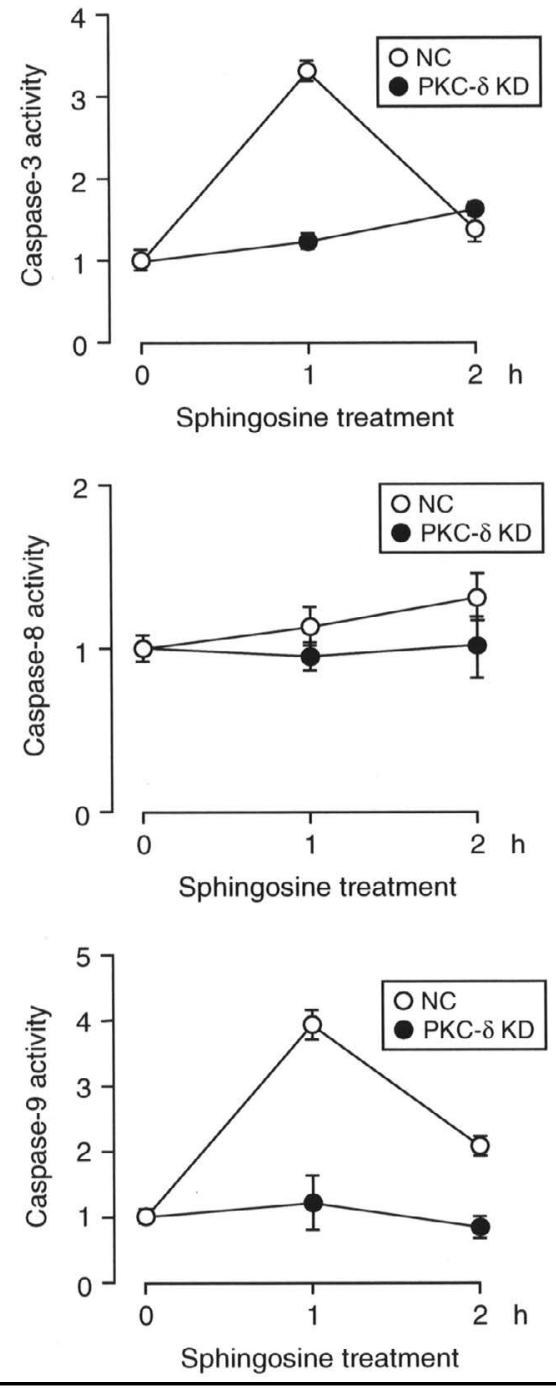

Fig. 6. Sphingosine-induced activation of caspase3/-9 in MKN-28 cells. Activities of caspase-3, -8, and -9 were enzymatically assayed in $\mathrm{MKN}-28$ cells transfected with the NC siRNA (NC) or the PKC$\delta$ siRNA (PKC- $\delta$ KD) before and after treatment with sphingosine $(100 \mu \mathrm{M})$. In the graphs, each point represents the mean $( \pm \mathrm{SEM})$ ratio against basal caspase activities (before treatment with sphingosine)( $\mathrm{n}=4$ independent experiments).

neither increase in SDK nor decrease in PKC- $\delta$ was obtained with MKN-45 cells (Fig. 3B). These results indicate that sphingosine promotes SDK production through proteolytic processing of PKC- $\delta$ in MKN-28 cells, but not MKN-45 cells.

14-3-3 protein is a target of SDK phosphorylation [4,5]. Sphingosine $(100 \mu \mathrm{M})$ increased phosphorylated 14-3-3 protein $\zeta$ in a treatment time (0-40 min)-dependent manner for 
MKN-28 cells (Fig. 4A), but such effect was not found with MKN-45 cells (Fig. 4B). This suggests that sphingosine binds to and activates SDK, to phosphorylate 14-3-3 protein.

Sphingosine disrupts mitochondrial membrane potentials, activates caspase-3/-9 in MKN28 cells in an SDK-dependent manner

Phosphorylated 14-3-3 protein could cause disruption of mitochondrial membrane potentials by dissociating Bax. To address this point, mitochondrial membrane potentials were monitored using DePsipher ${ }^{\mathrm{TM}}$, a mitochondrial activity marker. DePsipher ${ }^{\mathrm{TM}}$ is detected as an orange-red fluorescence at an absorbance of $590 \mathrm{~nm}$ for normal mitochondrial membrane potentials, but in case of the disruption the dye turns into green fluorescence at an absorbance of $530 \mathrm{~nm}$. For MKN-28 cells, the mitochondria exhibited orange-red fluorescent signals alone without green fluorescent signals (Fig. 5). Sphingosine (100 $\mu \mathrm{M})$ accumulated green fluorescent signals with weaker orange-red fluorescent signals (Fig. $5)$. This confirms that sphingosine disrupts mitochondrial membrane potentials in MKN28 cells. Sphingosine $(100 \mu \mathrm{M})$-induced disruption of mitochondrial membrane potentials was significantly inhibited by transfecting with the PKC- $\delta$ siRNA (Fig. 5). Collectively, these results indicate that sphingosine disrupts mitochondrial membrane potentials in an SDKdependent manner in MKN-28 cells.

Disruption of mitochondrial membrane potentials could allow cytochrome c efflux from the mitochondria, to form a complex with Apaf-1/dATP, causing activation of caspase-9 and the effector caspase-3. To answer this question, we enzymatically assayed activities of caspase-3, -8 , and -9 . Expectedly, sphingosine $(100 \mu \mathrm{M})$ activated caspase- 3 and -9 , but not caspase-8, for MKN-28 cells, with the peak $1 \mathrm{~h}$ after treatment (Fig. 6). Sphingosine (100 $\mu \mathrm{M}$ )-induced activation of caspase- 3 and -9 was clearly prevented by transfecting with the PKC- $\delta$ siRNA (Fig. 6). Sphingosine, thus, appears to activate caspase- 9 followed by caspase- 3 in concert with damaged mitochondria in an SDK-dependent manner.

\section{Discussion}

The present study examined the apoptotic effect of sphingosine on two types of human gastric cancer cell lines such as well differentiated MKN-28 cells and poorly differentiated MKN-45 cells. Sphingosine induced apoptosis in MKN-28 cells, with the potential much greater than for MKN-45 cells. Interestingly, sphingosine has the potential to inhibit PKC- $\delta$ [6]. This raises the possibility that sphingosine might induce apoptosis in MKN-28 cells by inhibiting PKC- $\delta$. The sphingosine effect here, however, was not affected by GF109203X, that inhibits PKCs including PKC- $\delta$ by binding to the ATP binding site on PKCs. This would rule out sphingosine-induced $\mathrm{MKN}-28$ cell apoptosis due to PKC- $\delta$ inhibition. In contrast, the sphingosine effect was prevented by transfecting with the PKC- $\delta$ siRNA, to decrease presence of SDK in association with decreased PKC- $\delta$ expression. This suggests that SDK, but not PKC- $\delta$, is implicated in sphingosine-induced MKN-28 cell apoptosis.

Sphingosine increased SDK in parallel with decreased PKC- $\delta$ for MKN-28 cells, but such effect was not found with MKN-45 cells. SDK is produced through proteolytic processing of PKC- $\delta[2]$. Sphingosine, accordingly, is likely to promote SDK production from PKC- $\delta$ and activate SDK in MKN-28 cells. SDK is recognized to specifically phosphorylate 14-3-3 protein $[4,5]$. In the present study, sphingosine increased phosphorylated 14-3-3 protein $\zeta$ in MKN28 cells, but not in MKN-45 cells. 14-3-3 protein inhibits apoptosis by capturing Bax [7]. Conversely, phosphorylated 14-3-3 protein dissociates Bax from a complex with 14-3-3 protein and in turn, free Bax moves towards the mitochondria and makes holes to disrupt mitochondrial membrane potentials, leading to mitochondrial apoptosis [8-11]. Sphingosine here disrupted mitochondrial membrane potentials in MKN-28 cells, and the effect was inhibited by transfecting with the PKC- $\delta$ siRNA. This suggests that sphingosine activates SDK, to phosphorylate 14-3-3 protein and disrupt mitochondrial membrane potentials in MKN-28 cells. 
Mitochondrial damage allows cytochrome c efflux from the mitochondria, which forms a complex with Apaf-1/dATP to activate caspase- 9 and the effector caspase-3. In the present study, sphingosine significantly activated caspase- 3 and -9 , but not caspase-8, in MKN-28 cells, and the activation was abolished by transfecting with the PKC- $\delta$ siRNA. This provides evidence that sphingosine activates caspase- 9 and the effector caspase- 3 in an SDK-dependent manner for MKN-28 cells. Overall, these results lead to a conclusion that sphingosine promotes SDK production from PKC- $\delta$ and activates SDK, to phosphorylate 143-3 protein, thereby dissociating Bax from a complex with 14-3-3 protein and consequently disrupting mitochondrial membrane potentials, to activate caspase- 9 followed by the effector caspase-3, responsible for MKN-28 cell apoptosis. Sphingosine induced apoptosis in MKN28 cells, with the potential much higher than for MKN-45 cells, and SDK for MKN-28 cells was produced more than for MKN-45 cells. This suggests that the potential for the apoptotic action of sphingosine may depend on SDK production.

In conclusion, the results of the present study show that sphingosine induces apoptosis in well differentiated MKN-28 human gastric cancer cells in an SDK-dependent manner.

\section{References}

1 Phillips DC, Martin S, Doyle BT, Houghton JA: Sphingosine-induced apoptosis in rhabdomyosarcoma cell lines is dependent on pre-mitochondrial Bax activation and post-mitochondrial caspases. Cancer Res 2007;67:756-764.

-2 Suzuki E, Handa K, Toledo MS, Hakomori S: Sphingosine-dependent apoptosis: a unified concept based on multiple mechanisms operating in concert. Proc Natl Acad Sci USA 2004;101:14788-14793.

- Kanno T, Nishizaki T: Sphingosine induces apoptosis in hippocampal neurons and astrocytes by activating caspase-3/-9 via a mitochondrial pathway linked to SDK/14-3-3 protein/Bax/cytochrome c. J Cell Physiol 2011;226:2329-2337.

4 Hamaguchi A, Suzuki E, Murayama K, Fujimura T, Hikita T, Iwabuchi K, Handa K, Withers DA, Masters SC, Fu $\mathrm{H}$, Hakomori S: A sphingosine-dependent protein kinase that specifically phosphorylates 14-3-3 (SDK1) is identified as the kinase domain of PKC $\delta$ : a preliminary note. Biochem Biophys Res Commun 2003;307:589594.

5 Hamaguchi A, Suzuki E, Murayama K, Fujimura T, Hikita T, Iwabuchi K, Handa K, Withers DA, Masters SC, Fu H, Hakomori S: Sphingosine-dependent protein kinase-1, directed to 14-3-3, is identified as the kinase domain of protein kinase C $\delta$. J Biol Chem 2003;278:41557-41565.

6 Keenan C, Goode N, Pears C: Isoform specificity of activators and inhibitors of protein kinase C $\gamma$ and $\delta$. FEBS Lett 1997;415:101-108.

7 Nomura M, Shimizu S, Sugiyama T, Narita M, Ito T, Matsuda H, Tsujimoto Y: 14-3-3 interacts directly with and negatively regulates pro-apoptotic Bax. J Biol Chem 2003;278:2058-2065.

8 Rosenquist M, Sehnke P, Ferl RJ, Sommarin M, Larsson C: Evolution of the 14-3-3 protein family: does the large number of isoforms in multicellular organisms reflect functional specificity? J Mol Evol 2000;51:446458.

-9 Shen YH, Godlewski J, Bronisz A, Zhu J, Comb MJ, Avruch J, Tzivion G: Significance of 14-3-3 selfdimerization for phosphorylation-dependent target binding. Mol Biol Cell 2003;14:4721-4733.

10 Tsuruta F, Sunayama J, Mori Y, Hattori S, Shimizu S, Tsujimoto Y, Yoshioka K, Masuyama N, Gotoh Y: JNK promotes Bax translocation to mitochondria through phosphorylation of 14-3-3 proteins. EMBO J 2004;23:1889-1899.

11 Woodcock JM, Murphy J, Stomski FC, Berndt MC, Lopez AF: The dimeric versus monomeric status of 14-3$3 \zeta$ is controlled by phosphorylation of Ser58 at the dimer interface. J Biol Chem 2003;278:36323-36327. 